\title{
Optical Manipulation with Random Light Fields: From Fundamental Physics to Applications
}

\author{
Giorgio Volpe $^{1 *}$, Sylvain Gigan ${ }^{2}$, and Giovanni Volpe ${ }^{3,4}$ \\ ${ }^{1}$ Department of Chemistry, University College London, 20 Gordon Street, London WC1H 0AJ, United Kingdom \\ ${ }^{2}$ Laboratoire Kastler Brossel, UMR8552 of CNRS and Université Pierre et Marie Curie, 24 rue Lhomond, 75005 Paris, France \\ ${ }^{3}$ Soft Matter Lab, Physics Department, Bilkent University, Cankaya, 06800 Ankara, Turkey \\ ${ }^{4} U N A M$ - National Nanotechnology Research Center, Bilkent University, Cankaya, 06800 Ankara, Turkey \\ *g.volpe@ucl.ac.uk
}

\begin{abstract}
Speckles are random light fields that share some universal statistical properties. Because of this, they can be used to perform deterministic optical manipulation tasks on a Brownian particle as well as control its diffusion properties.

OCIS codes: (030.6140) Speckle; (170.4520) Optical confinement and manipulation; (350.4855) Optical tweezers or optical manipulation.
\end{abstract}

\section{Introduction}

Optical tweezers have been widely applied to non-invasively manipulate micro- and nano-objects. They have, therefore, gained increasing importance as tools in microbiology and biophysics both for fundamental studies and for more advanced applications, such as optical sorting and optical delivery [1-2].

Most of current optical manipulation techniques, however, rely either on carefully engineered optical systems or advanced fabrication tools. Although similar conditions are routinely met in research laboratories, these requirements can be very stringent and limit the applicability of these techniques, for example, to biomedical and microfluidic applications, where simplicity, low-cost and high-throughput are key aspects.

One more challenge common to all these techniques in optical manipulation is the light scattering occurring in optically complex media, such as biological tissues, turbid liquids and rough surfaces, which naturally gives rise to apparently random light fields known as speckles [3].

Here, we show how the statistical properties of speckle light fields can be used to transform them into a versatile tool to efficiently perform fundamental optical manipulation tasks such as trapping, guiding and sorting. Similarly, we show how speckle light fields can be used to control and tune the diffusion property of a Brownian particle, becoming an ideal model system to study the motion of particles in random potentials [4-5].

\section{Optical forces in speckle light fields}

Speckle patterns are complex interference patterns that can be generated by different processes, such as scattering of a laser on a rough surface, multiple scattering in an optically complex medium, or mode-mixing in a multimode optical fiber [3]. In general, they are the result of the interference of a large number of waves propagating along different directions and with a random phase distribution, and, despite their random appearance, they share some universal statistical properties. In particular, a speckle pattern has a negative exponential intensity distribution and the normalized spatial autocorrelation function can be approximated by a Gaussian [4].

The motion of a Brownian particle in a speckle field is the result of random thermal forces and deterministic optical forces. Optical gradient forces are the dominant deterministic forces acting on dielectric particles whose size is comparable or smaller than the average speckle grain as defined by the speckle spatial autocorrelation function, and they attract particles with high-refractive index towards the intensity maxima of the optical field. As a particle moves in the speckle, the optical force acting on it changes both in magnitude and direction with a characteristic time scale that in first approximation is inversely proportional to the average speckle intensity. The emergence of behaviours, such as the possibility of tuning the anomalous diffusion of a Brownian particle or of performing deterministic optical manipulation tasks on it, is controlled by this characteristic time [4-5].

[1] O. M. Maragò, P. H. Jones, P. G. Gucciardi, G. Volpe and A. C. Ferrari, “Optical trapping and manipulation of nanostructures," Nature Nanotech. 8, 807-819 (2013).

[2] G. Volpe and G. Volpe, "Simulation of a Brownian particle in an optical trap," Am. J. Phys. 81, 224-230 (2013).

[3] A. P. Mosk, A. Lagendijk, G. Lerosey and M. Fink, "Controlling waves in space and time for imaging and focusing in complex media," Nature Photon. 6, 283-292 (2012).

[4] G. Volpe, G. Volpe, S. Gigan, "Brownian Motion in a Speckle Light Field: Tunable Anomalous Diffusion and Selective Optical Manipulation," Sci. Rep. 4, 3936 (2014).

[5] G. Volpe, L. Kurz, A. Callegari, G. Volpe, S. Gigan, "Speckle Optical Tweezers: micromanipulation with random light fields," Opt. Express 22, 18159-18167 (2014). 